\title{
Intrathecal Low Dose Bupivacaine with Fentanyl or Clonidine in TURP Surgery: A Prospective Randomized Double Blind Study
}

\author{
Lalit K. Raiger ${ }^{1}$, Sandeep Sharma' ${ }^{2}$ Pramod Jain ${ }^{3}$, Shailendra Pareek ${ }^{4}$ \\ ${ }^{1}$ Sr. Professor, Department of Anaesthesiology, RNT Medical College, Udaipur, Rajasthan, India, ${ }^{2}$ Assistant Professor, Department of Anaesthesiology, \\ RNT Medical College, Udaipur, Rajasthan, India, ${ }^{3}$ Senior specialist, District Hospital, Alwar, Rajasthan, India, ${ }^{4}$ Consultant, Narayana Hrudayalaya, Jaipur, \\ Rajasthan, India
}

\section{Abstract}

Background: Present study was designed to compare the effect of intrathecal clonidine and fentanyl in combination with low doses of bupivacaine in TURP surgery on the hemodynamics, quality of sensory and motor block as well as the duration of analgesia and motor block. Subjects and Methods: 120 patients scheduled to undergo TURP surgery were randomized into four groups. Group I and II received hyperbaric bupivacaine $5 \mathrm{mg}$ and $7.5 \mathrm{mg}$ respectively with fentanyl $25 \mu \mathrm{g}$ whereas Group III and IV received Bupivacaine $5 \mathrm{mg}$ and $7.5 \mathrm{mg}$ respectively with clonidine $30 \mu \mathrm{g}$ in subarachnoid space. Hemodynamic parameters (heart rate, systolic blood pressure, diastolic blood pressure) were recorded at regular intervals. Intensity of motor block was recorded at L2 to S1 myotomes and a myotome score was calculated for each limb. Measurement of level of pinprick analgesia and intensity of motor block were recorded at 0,2,4,8 and 10 minutes. Time to return of pain sensation and complete motor recovery was also noted. Results: There was statistically significant difference in occurance of bradycardia from baseline in group I at 10 and 20 minutes $(\mathrm{p}<0.05, \mathrm{p}<0.001)$. In group IV there was significant fall in heart rate at 10,20 and 30 minutes ( $<<0.05, p<0.05, p<0.001)$. In group II and III there was no significant fall in heart rate. In group I there is significant fall in SBP at 10,20 and 30 minutes $(\mathrm{p}<0.05)$. In group II there is significant fall in SBP at 10 and 20 minute ( $<<0.05$ ). In group III and IV, there is significant fall in SBP at 10, 20 and 30 minutes $(\mathrm{p}<0.05, \mathrm{p}<0.05, \mathrm{p}<0.001)$. The maximum level of sensory block was comparable in all the groups (T10.21 $\pm 0.99, \mathrm{~T} 10.30 \pm 0.75$, T10.33 \pm 1.12 , T10.13 \pm 1.11 in groups I,II,III and IV respectively). There is no statistical significant difference in achieving level of motor block on comparing group I/II and group III/IV but there is highly significant $(p<0.001)$ difference in group I/III, group I/IV, group II/III, group II/IV at 10 minutes after onset of block. There was no statistical significant difference in return of pain sensation in all groups $(91.60 \pm 18.95,86.07 \pm 10.84,85.37 \pm 17.39,85.50 \pm 22.22$ in groups I,II,III and IV respectively). Complete return of motor power (modified Bromage score $=0$ ) was seen at $158.17 \pm 18.17 \mathrm{~min}, 154 \pm 18.50 \mathrm{~min}, 146.53 \pm 16.54 \mathrm{~min}$ and $154.83 \pm 21.27 \mathrm{~min}$ in group I,II,III and IV respectively. There is significant difference $(\mathrm{p}<0.05)$ in return of motor power on comparing group I with III, otherwise all groups are comparable. Conclusion: The addition of fentanyl or clonidine to intrathecal bupivacaine for TURP does not result in any significant difference in the quality and intensity of sensory block. Clonidine causes a greater degree of motor block when compared to fentanyl. Both clonidine and fentanyl causes bradycardia and hypotension although the degree and duration of hypotension is greater with clonidine.

Keywords: Hyperbaric bupivacaine, Clonidine, Fentanyl, Myotome score, Modified bromage score.

Corresponding Author: Dr. Sandeep Sharma, Assistant. Professor, Department of Anaesthesiology, RNT Medical College, Udaipur, Rajasthan, India.

Received: December 2018

Accepted: January 2019

\section{Introduction}

Transurethral resection of prostate (TURP) was originated by Guthrie in 1934 and developed by McCarthy in 1931. TURP is a cystoscopic procedure used to alleviate the symptoms of bladder outflow obstruction usually caused by benign prostatic hyperplasia (BPH). This procedure is most commonly performed on elderly patients, a population with a high incidence of cardiac, pulmonary and renal disease.

Spinal anesthesia has been widely used for urological operation. However, the decrease in CSF volume is responsible for the higher anesthetic level achieved in the geriatric population for a given dose of local anesthesia.
Failure to reduce standard dose in the elderly may produce a high sensory and motor block. Low dose bupivacaine used alone decreases the incidence of high spinal block but could not provide adequate level of sensory block.

These observations generated interest in an alternative adjuvant to reduce the dose of local anesthetic along with prolonging motor and sympathetic blockade without delaying recovery. In present study, we compared the effect of clonidine and fentanyl in combination with low doses of hyperbaric bupivacaine in subarachnoid block on the hemodyamics, quality of sensory and motor block as well as the duration of analgesia and motor block. 


\section{Subjects and Methods}

After hospital ethical committee approval, this study was conducted in Government MB hospital associated with RNT Medical College, Udaipur.

\section{Sample Size}

Based on a previous study by Sethi et all we obtained that a sample size of 58 patients distributed in two groups was required for the study to have an $\alpha$-error of 0.05 and a $\beta$-error of >0.9. To compensate for dropouts each group was assigned with 30 patients.

\section{Statistical Analysis}

Data were analysed using SPSS (version 24.0). All data were expressed as mean \pm SD. normally distributed data were subjected to student's t test and $\mathrm{p}$ value of 0.05 was considered significant.

\section{Study Design}

120 patients scheduled to undergo TURP surgery were randomized using opaque sealed envelop technique into four groups (30 patients in each group).

Group I -Hyperbaric bupivacaine $5 \mathrm{mg}$ with fentanyl $25 \mu \mathrm{g}$ Group II - Hyperbaric bupivacaine $7.5 \mathrm{mg}$ with fentanyl 25 $\mu \mathrm{g}$

Group III - Hyperbaric bupivacaine $5 \mathrm{mg}$ with clonidine 30 $\mu \mathrm{g}$

Group IV- Hyperbaric bupivacaine $7.5 \mathrm{mg}$ with clonidine 30 $\mu \mathrm{g}$

The final volume was adjusted to $2.0 \mathrm{ml}$ by using normal saline.

\section{Exclusion Criteria}

Patients with deformities of the spinal column, post spine surgeries, psychiatric illness, history of allergy to study drugs, cardiovascular disease, renal disease, respiratory disease, neurological disorders, coagulopathies and refusal for spinal anesthesia were excluded from the study.

\section{Pre-Surgical Protocol}

The day prior to surgery all patients were asked to undergo a detailed pre-anaesthetic evaluation and all were advised to fast the night prior to surgery and received tablet alprazolam $0.25 \mathrm{mg}$ and tablet ranitidine $150 \mathrm{mg}$ orally on the previous night and the morning of surgery.

\section{Surgical Protocol}

On day of surgery, a written informed consent was obtained from each patient. On arrival in OT, routine non-invasive monitoring was applied, and vital signs were monitored with electrocardiography, pulse oximetry and automated oscilliotonometry. Intravenous access was secured with 18G IV cannula, and infusion of Ringer's lactate solution started. Spinal anesthesia was administered at the L3-L4 interspace with the patient in sitting position by using a 25 gauge Quincke needle after confirming free flow of cerebrospinal fluid. The same anesthesiologist performed all the blocks, who was blind to the drug used.
Vitals (heart rate, systolic blood pressure and diastolic blood pressure) were recorded at 5,10,20 and 30 minutes. Hypotension (systolic arterial pressure $<90 \mathrm{mmHg}$ or $>10 \%$ decrease from the baseline) was treated with $6 \mathrm{mg}$ incremental dose of mephenterine intravenously.

Bradycardia (heart rate $<60 \mathrm{bpm}$ or $<10 \%$ from the initial value) was treated with intravenous atropine $0.3 \mathrm{mg}$.

When the patient demanded the need for additional analgesia, tramadol $2 \mathrm{mg} / \mathrm{kg}$ was given intravenously.

The level of sensory block was defined as the loss of sharp sensation by using a pinprick test recorded bilaterally, at the midclavicular line every 2 minutes till the three consecutive readings are same.

Motor block in the lower limb was assessed with references to specific myotomes. It was assessed by testing the strength and movements of the lower limb muscle according to the following five myotomes.

L2 - Hip flexion

L3 - Knee flexion

L4 - Ankle dorsiflexion

L5 - Great toe dorsiflexion

S1- Ankle planter flexion

Complete motor block or absent power at the above mentioned myotome and intensity of motor block was recorded. A myotome score, which is the number of myotomes blocked, from 0 to maximal 10 where score of 0 denotes complete motor block. The total score was calculated for each limb, the maximum score being 5 points for one side, 10 points in total.

Measurement of motor block was done at $0,2,4,8$ and 10 minutes. The time taken to return of complete pain sensation and complete return of motor power (modified bromage score of $0 / 3$ ) was also noted [Table 1]. The patient was discharged from the recovery room when the motor block was completely resolved.

Table 1: Modified Bromage Scale (Intensity of Motor Block)
\begin{tabular}{|l|l|l|}
\hline Scale & Criteria & Degree of Block \\
\hline 0 & $\begin{array}{l}\text { Free movement of leg and feet, able to } \\
\text { raise extended leg }\end{array}$ & None \\
\hline 1 & $\begin{array}{l}\text { Inability to raise extended leg, knee flexion } \\
\text { decreased, full flexion of feet and ankle }\end{array}$ & Partial 33\% \\
\hline 2 & $\begin{array}{l}\text { Inability to raise leg or flex knees; flexion } \\
\text { of ankle and feet present }\end{array}$ & Partial 66\% \\
\hline 3 & $\begin{array}{l}\text { Inability to raise leg, flex knee or ankle or } \\
\text { move toes }\end{array}$ & Complete \\
\hline
\end{tabular}

Respiratory depression (respiratory rate of $\leq 8$ breaths/min and/ or oxygen saturation $\leq 85 \%$ in room air), and other adverse effects including pruritus, nausea, vomiting, shivering were also recorded.

\section{Results}

There was no statistically significant difference in demographic distribution (age, gender, weight) among the groups. ASA grade of patients was also comparable in all the groups with no statistically significant difference.

Heart Rate (occurrence of bradycardia): There was statistical significant difference in fall of heart rate from baseline in group I at 10 minute $(\mathrm{p}<0.05)$ and highly significant fall at 
20 minute $(\mathrm{p}<0.001)$. In group IV there was significant fall in heart rate at 10 minute and 20 minute $(\mathrm{P}<0.05)$ and highly significant fall in heart rate at 30 minute $(\mathrm{p}<0.001)$. In group II and III there was no significant fall in heart rate [Figure 1]. Systolic Blood Pressure (SBP): In group I there was significant fall in SBP at 10, 20 and 30 minutes $(\mathrm{p}<0.05)$. In group II there was significant fall in SBP at 10 and 20 minute $(\mathrm{p}<0.05)$. In group III there was significant fall in SBP at 5 and 10 minutes $(\mathrm{P}<0.05)$ and highly significant fall in $\mathrm{BP}$ at 20 and 30 minutes $(\mathrm{p}<0.001)$. In group IV there was significant fall in SBP at 10, 20 minutes $(\mathrm{P}<0.05)$ and highly significant fall at 30 minutes $(\mathrm{p}<0.001)$ [Figure 2].

Diastolic Blood Pressure (DBP): In group I there was significant fall in DBP at 30 minutes $(\mathrm{p}<0.05)$ and highly significant fall at 20 minutes $(\mathrm{p}<0.001)$. In group II there was significant fall in DBP at 5 minute $(\mathrm{p}<0.05)$ and highly significant fall at 10,20 and 30 minutes $(p<0.001)$. In group III there was significant fall in DBP at 10 and 30 minutes $(\mathrm{P}<0.05)$. In group IV there was significant fall in DBP at 5, 10 and 30 minutes $(\mathrm{P}<0.05)$ and highly significant fall in DBP at 20 minutes $(\mathrm{p}<0.001)$.

Level of Sensory Block: There was no statistical difference in reaching maximum level of sensory block in all four groups, so all groups are comparable (T10.21 \pm 0.99 , $\mathrm{T} 10.30 \pm 0.75, \mathrm{~T} 10.33 \pm 1.12, \mathrm{~T} 10.13 \pm 1.11$ in group I,II,III and IV respectively) [Figure 3].

Level of Motor Block (Left Lower Limb): In statistical analysis of number of myotomes blocked on left side, there was no statistical difference in group I/II and group III/IV but there was highly significant $(\mathrm{p}<0.001)$ difference in group I/III, group I/IV, group II/III, group II/IV at 10 minutes after onset of block on left side [Figure 4].

Level of Motor Block (Right Lower Limb):There was no statistical difference in number of myotomes blocked on right side in group I/II and group III/IV but there was highly significant $(\mathrm{p}<0.001)$ difference in group I/III, group I/IV, group II/III, group II/IV. There was significant difference $(p<0.05)$ in level of motor block on right side in group III/IV at 4 minutes also [Figure 4].

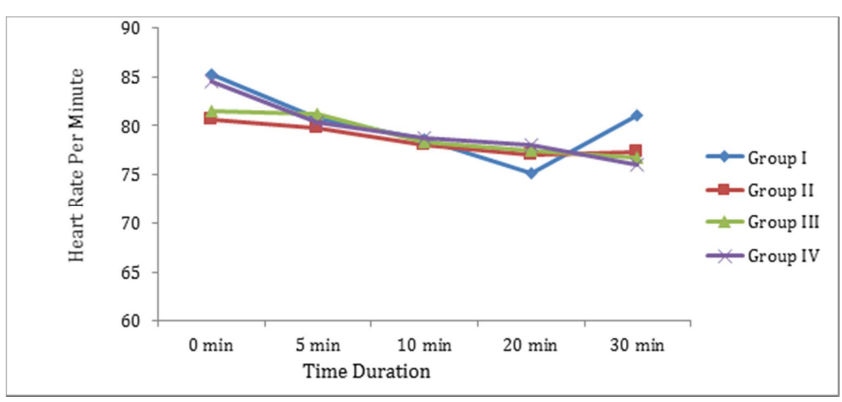

Figure 1: Changes in heart rate

Return of pain sensation: There was no statistical significant difference observed in return of pain sensation in all groups $(91.60 \pm 18.95, \quad 86.07 \pm 10.84,85.37 \pm 17.39, \quad 85.50 \pm 22.22$ in groups I,II,III and IV respectively) [Table 1]

Return of motor power: Complete return of motor power (modified Bromage score $=0$ ) was seen at $158.17 \pm 18.17 \mathrm{~min}$, $154 \pm 18.50 \mathrm{~min}, 146.53 \pm 16.54 \mathrm{~min}$ and $154.83 \pm 21.27$ min in group I,II,III and IV respectively. There is significant difference $(p<0.05)$ in return of motor power on comparing group I with III, otherwise all groups are comparable
[Table 1].

There was no significant difference in occurrence of adverse effects (nausea, vomiting, pruritus, hypotension, shivering, bradycardia, respiratory depression) in all the groups.

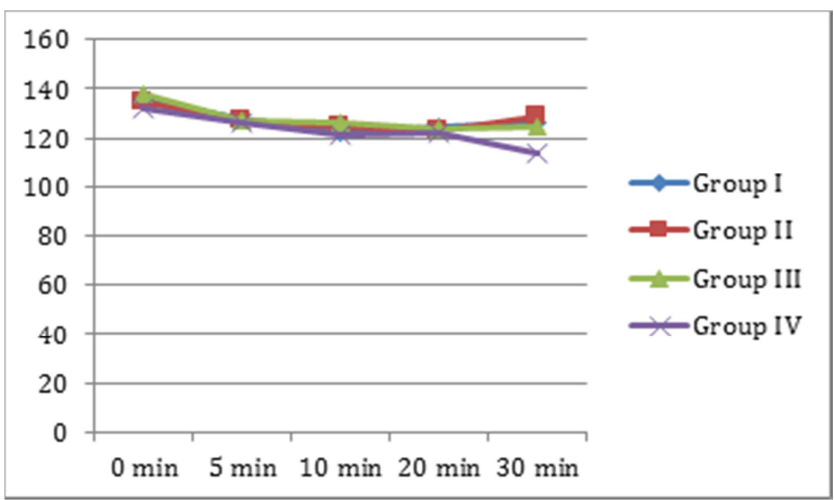

Figure 2: Changes in SBP

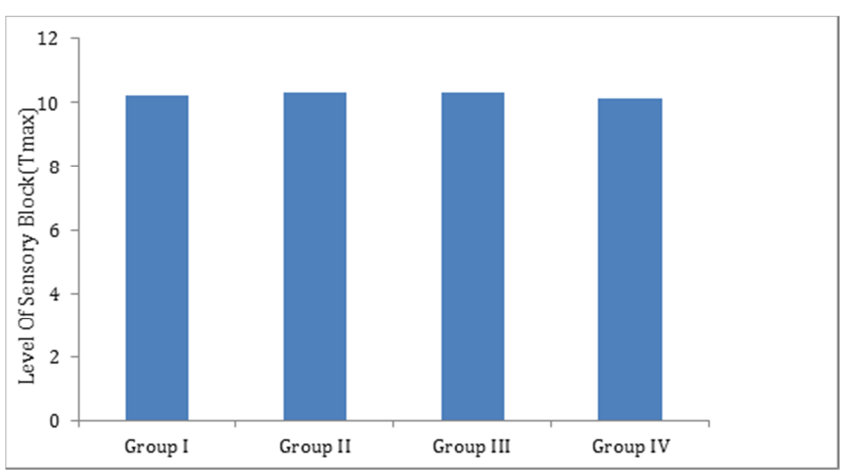

Figure 3: Comparison of level of sensory block between groups

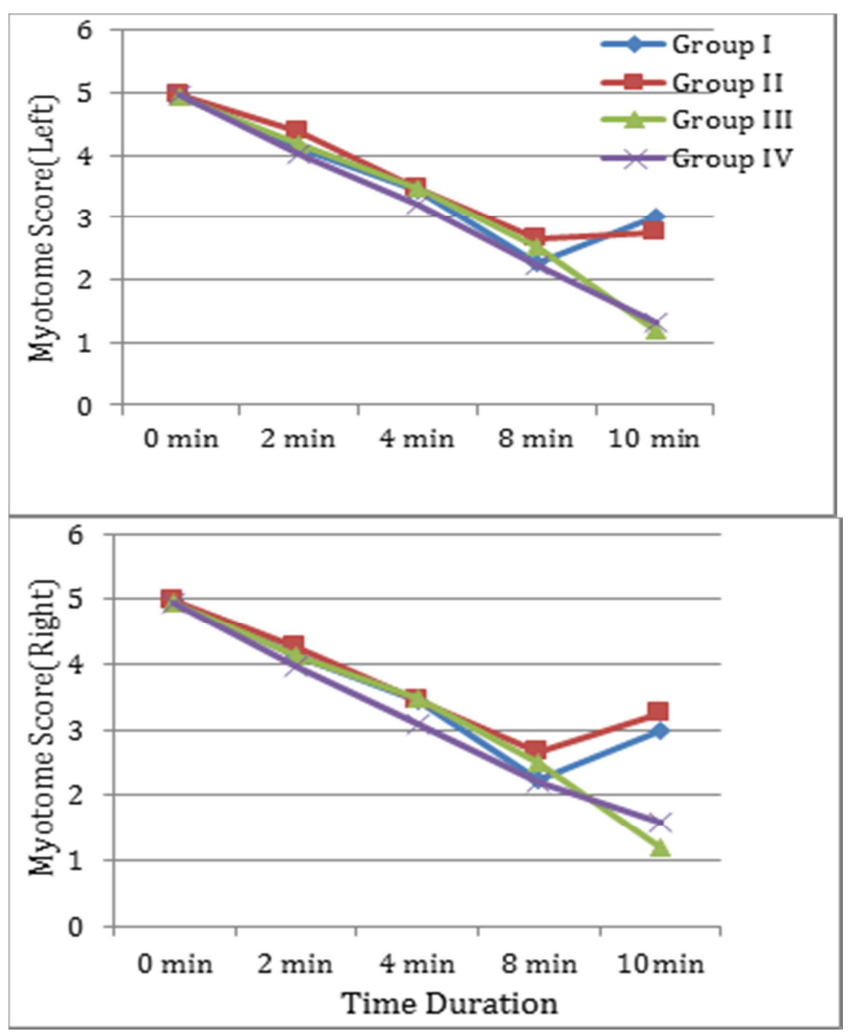

Figure 4: Myotome Score 
Table 1: Return of pain sensation and motor power

\begin{tabular}{|l|l|l|}
\hline Group & $\begin{array}{l}\text { Pain } \\
\text { (min)* }\end{array}$ & $\begin{array}{l}\text { Return of Motor } \\
\text { Power (min)* }\end{array}$ \\
\hline Group I & $91.60 \pm 18.95$ & $158.17 \pm 18.17$ \\
\hline Group II & $86.07 \pm 10.84$ & $154.83 \pm 18.50$ \\
\hline Group III & $85.37 \pm 17.39$ & $146.53 \pm 16.54$ \\
\hline Group IV & $85.50 \pm 22.22$ & $154.83 \pm 21.27$ \\
\hline *Mean \pm SD
\end{tabular}

\section{Discussion}

Regional anesthesia has long been considered the anesthetic technique of choice for transurethral resection of prostate (TURP) and is used in more than $70 \%$ of these procedure worldwide. ${ }^{[2]}$ It allows the patient remain awake thus helping in the early diagnosis of transurethral resection of prostate (TURP) syndrome.

Further, it confines additional advantages such as decreased blood loss, decreased requirement of analgesics postoperatively, improve cognitive function. ${ }^{[3-5]}$ When spinal anesthesia is used for the procedure, a T10 dermatome anesthetic level is needed to perform the procedure comfortably and safely, however majority of patient undergoing TURP are elderly and are likely to have compromised cardiopulmonary reserves. Therefore, it is extremely important to limit the distribution of block in order to reduce adverse hemodynamic and pulmonary effects in such patients.

In this study we observed statistically significant occurrence of bradycardia at 10,20 minutes in group I and at 10, 20 and 30 minutes in group IV and found that there was no significant bradycardia in group II and III at any given period of observation.

Kuusniemi et al, ${ }^{[6]}$ conducted study on the use of bupivacaine and fentanyl for spinal anesthesia for urologic surgery and reported incidence of bradycardia in 3 patients out of total 80 patients who received intrathecal bupivacaine and fentanyl, however their investigation defined bradycardia as heart rate of less than 50 beats/minute or decreased more than $20 \%$ from baseline. The present study used more stringent criteria for bradycardia ( $>10 \%$ fall from baseline). This may explain the more frequent occurrence of bradycardia in the same.

Several other investigators including Ishwar Singh et al, ${ }^{[7]} \mathrm{D}$ Benhamou had evaluated fentanyl as an additive to intrathecal hyperbaric bupivacaine and had reported insignificant incidences of bradycardia. However they had use definition of bradycardia as less than 60 beats per minutes as opposed to the definition used in the present study. ${ }^{[8]}$

In present study the occurrence of bradycardia in group I and group IV as against no bradycardia in groups II and III suggest that there are other reasons besides the intrathecally administered drug for the same. Prominent amongst these reasons might be the absence of uniform protocol for pre loading each patient with the same amount of fluid prior to the administration of the block. Several studies have suggested that the volume and type of preload would greatly affect hemodynamics in patient undergoing TURP surgery. ${ }^{[9-}$ 11] Apart from this the amount of intra operative blood loss, irrigation fluid used and lithotomy position would also have bearing on intraoperative heart rate and the subsequent occurrence or absence of bradycardia.

In the present study, there was a significant fall in systolic blood pressure(SBP) in all the 4 groups at 10 and 20 minutes. In addition there was a highly significant fall in SBP in group III and IV in which clonidine was used as an additive. Similarly there was a significant fall in diastolic blood pressure in group I at 20 and 30 minutes; in group III at 10 and 30 minutes and in group II and IV at 5,10,20 and 30 minutes.

Intrathecally administered bupivacaine causes central sympatholysis by virtue of its action on efferent sympathetic nerves arising out of the spinal cord, specially in the mid and lower thoracic spinal cord. This causes a loss of vascular tone. Since the vascular tone is the primary element determining diastolic B.P, the fall in diastolic blood pressure was more in groups II and IV in which higher doses of bupivacaine $(7.5 \mathrm{mg})$ were used, as compared to group I and III (5mg).

Fentanyl causes hypotension by direct myocardial depressant activity, while clonidine causes central sympatholysis by inhibitory sympathetic outflow.

Ishwar Singh et al, ${ }^{[7]}$ conducted a study on 50 patients that underwent elective lower abdominal, lower limb and urological procedure under spinal anaesthesia using bupivacaine with fentanyl and sufentanil. They did not report any significance occurrence of hypotension in either group, however they had defined hypotension as more than $20 \%$ decrease in systolic B.P. from baseline as opposed to the criteria of a fall more than $10 \%$ from baseline used in present study. Similar results were reported by Kim et al, ${ }^{[12]}$ using even broader definitions of hypotension as decreased in S.B.P. of less than $90 \mathrm{~mm}$ of $\mathrm{Hg}$ or less than $25 \%$ from baseline.

The present study evaluated the intra group occurrence of hypotension and bradycardia in patients receiving different dose of bupivacaine fentanyl and bupivacaine clonidine combination intrathecally for TURP surgery. All prior studies have concentrated on the comparative hemodynamic intergroup changes. This stresses the need to further evaluate the degree and duration of hemodynamic changes that occur within each group and their clinical significance. Moreover, this also highlights the fact that none of these combinations can be considered totally immune to hemodynamic disturbances, though some might be more resilient than the others.

The mean myotome score on left lower limb was significantly less in group III $(1.20 \pm 1.30)$ as opposed to group $\mathrm{I}(3.00 \pm 0.82)$ at $10 \mathrm{~min}$. This score was also significantly less than that of patients in group II $(2.75 \pm 0.50)$. The myotome score was also significantly less in group IV $(1.33 \pm 0.82)$ as compared to group I $(3.00 \pm 0.82)$ and group II $(2.75 \pm 0.50)$. Similarly the myotome score on the right lower limb was also significantly less in group III(1.20 \pm 1.30$)$ as compared to group I $(3.0 \pm 0.82)$ and group $\mathrm{II}(3.25 \pm 0.50)$ at 10 minutes and in group IV $(1.57 \pm 0.53)$ as compared to group I and II. Thus the motor blockade achieved by group III was significantly greater in both lower limbs as compared to other groups.

The present study had shown that increasing the dose of bupivacaine does not increase the motor blockade (group I $\mathrm{v} / \mathrm{s}$ group II and group III v/s group IV). This suggests that 
the additive effect of clonidine/ fentanyl would potentiate the block more than that caused by increased dose of bupivacaine.

Present study showed that while the level of sensory blockade achieved by any of the four combination did not varies significantly, the level of motor block was greater in patients who received clonidine as an adjuvant and was also dependent on the dose of local anesthetic. Several studies have found that clonidine enhances the degree of motor blockade when added to bupivacaine for SAB. ${ }^{[13]}$ A similar investigation tract compared the effect of adding 15 and 30 $\mu \mathrm{g}$ of clonidine to bupivacaine for inguinal herniorrhaphy and found increased motor block with clonidine as compared to plain bupivacaine. Juitaet al, ${ }^{[13]}$ also reported a significantly prolonged anaesthesia time with bromage score 2 for clonidine groups compared to sufentanil groups in gynaecological surgery. Routray et al, ${ }^{[14]}$ had also reported a increased duration of motor block in clonidine group as compared to fentanyl group when administerd intrathecallywith hyperbaric bupivacaine in lower limb orthopaedic surgeries. The fact that present study demonstrates the motor block enhancing effect of clonidine in doses as low as $30 \mu \mathrm{g}$ is also of importance as a majority of earlier studies have showed this effect only at doses greater than $50 \mu \mathrm{g}$; therefore it is important to realize that even low doses of intrathecal clonidine can cause a undesirably high motor block. ${ }^{[15,16,3]}$ The explanation for this could be that the alpha-2 adrenoceptor agonist induce cellular modification in the ventral horn of the spinal cord (Motor neuron hyper polarization) and facilitate the local anesthetic action.

The use of adjuvants in subarachnoid block is well established. We have used the present study to determine whether the addition of clonidine as an adjuvant would provide any benefit than the more popular fentanyl. The study suggests that clonidine causes more hypotension and a greater degree of motor block than fentanyl. This would therefore favour the use of fentanyl as an additive.

There were no clinically significant occurrence of adverse effects in the groups studied.

\section{Conclusion}

1. The addition of both clonidine and fentanyl to local anesthetic for TURP results in bradycardia and hypotension. Although the degree and duration of hypotension is greater with clonidine as compared to fentanyl.

2. The addition of fentanyl or clonidine to bupivacaine for TURP does not result in any significant difference in the quality and intensity of sensory block.

3. Clonidine causes a greater degree of motor block when compared to fentanyl.

\section{References}

1. Sethi BS, Samuel M, Sreevastava D. Efficacy of analgesic effects of low dose intrathecal clonidine as adjuvant to bupivacaine. Indian Journal of Anaesthesia 2007;51(5):415-419

2. Raj PP, Gesund P,Pheruj. Position and choice for surgical procedure. Clinical Practice of R.A.P. 197, New York; Churchill Living Stone 1991.

3. Bowman GW, Hooth JW, Mcglothlen JS: Anesthesia for transurethral resection of the prostate: spinal or general? AANA J 1981;49(1):63-68

4. Chung FF, Chung A, Meier RH, Lautenschlaeger E, Seyone C: Comparison of preoperative mental function after general anesthesia and spinal anesthesia with intravenous sedation. Can J Anesth 1989:36:382

5. Mackenzie AR: Influence of anesthesia on blood loss in transurethral prostatectomy. Scot Med J 1990;35(1)14-16

6. Kuusniemi KS, Pihlajamaki KK, Pitkanen MT, Helenius HY, Kirvela A. The use of bupivacaine and fentanyl for spinal anesthesia for urologic surgery. Anesth Analg 2000;91:1452-1456

7. Singh I, Gupta M, Mahavar B,Gupta A. Comparison of effect of intrathecal sufentanil bupivacaine and fentanyl bupivacaine combination on postoperative analgesia. Indian Journal of Anaesthesia 2008:52(3):301-04

8. Benhamou D, Thorin D, Brichant JF, Dailland P, Milon D, Schneider $M$. Intrathecal clonidine and fentanyl with hyperbaric bupivacaine improves analgesia during caesarean section. Anesth Analg 1998;87:609-13

9. Duman A, Apiliogullari S: Patients undergoing TURP should receive the same amount of intravenous fluids. Anesth Analg 2009;109:287-8

10. Riesmeier A, Schellhaass A, Bold TJ, Suttner S. Crystalloid colloid versus crystalloid intravascular volume administration before spinal anesthesia in elderly patients: the influence on cardiac output and stroke volume. Anesth Analg 2009;108:650-4

11. Riley ET, Cohen SE, Robenstein AJ, Flanajan B. Prevention of hypotension after spinal anesthesia for caesarean section; six percent hetastarch versus lactated ringer's solution. Anesth Analg 1995; 81:83842

12. Kim SY, Cho JE, Hong JY, Koo BN, Kim JM. Comparison of intrathecal fentanyl and sufentanil in low dose dilute bupivacaine spinal anaesthesia for transurethral prostatectomy. Brit $\mathrm{J}$ Anesthesia 2009;103(5):750-4

13. Juilao MC, Lauretti GR. Low dose intrathecal clonidine combined with sufentanil as analgesic drugs in abdominal gynecological surgery. J Clin Anesth 2000; Vol 12(5):357-62

14. Routray SS, Raut K, Pradhan A, Dash A, Soren M. Comparison of intrathecal clonidine and fentanyl as adjuvant to hyperbaric bupivacaine in subarachnoid block for lower limb orthopedic surgery. Anesthesia essays and researches 2017;11 (3) : 589-593

15. Niemi L. Effects of intrathecal clonidine on duration of bupivacaine spinal anesthesia, hemodynamics and postoperative analgesia in patients undergoing knee arthroscopy. Acta Anesthesiol Scand 1994;38:724-80

16. Racle JP, Benkhadra A, Poy JY, Gleizal B. Prolongation of isobaric bupivacaine spinal anesthesia with epinephrine and clonidine for hip surgery in the elderly. Anesth Analg 1987;66:442-6

Copyright: () the author(s), publisher. Academia Anesthesiologica International is an Official Publication of "Society for Health Care \& Research Development". It is an open-access article distributed under the terms of the Creative Commons Attribution Non-Commercial License, which permits unrestricted non-commercial use, distribution, and reproduction in any medium, provided the original work is properly cited.

How to cite this article: Raiger LK, Sharma S, Jain P, Pareek S. Intrathecal Low Dose Bupivacaine with Fentanyl or Clonidine in TURP Surgery: A Prospective Randomized Double Blind Study. Acad. Anesthesiol. Int. 2018;3(2):13-17.

DOI: dx.doi.org/10.21276/aan.2018.3.2.3

Source of Support: Nil, Conflict of Interest: None declared. 